\title{
Die Ukrainer und der „Große Vaterländische Krieg“" Die Komplexität der Kriegsbiographien
}

Die Aufarbeitung der Geschichte des Zweiten Weltkriegs, der deutschen Besatzung und der Nachkriegszeit ist in der Ukraine heute deutlich weiter fortgeschritten als beispielsweise in Russland oder Belarus', was wohl auch darauf zurückzuführen ist, dass die Mythisierung des Sieges hier nicht so dominant ist, sondern sich mit vielfältigen anderen Erinnerungskulturen überlagert. Von ukrainischen Historikerinnen und Historikern sind in den letzten Jahren zahlreiche Impulse ausgegangen, wobei sie zum Teil an Arbeiten westeuropäischer und nordamerikanischer Historiker anknüpfen konnten. ${ }^{1}$ Auch der Zugang zu Archivquellen zur Kriegs- und Nachkriegszeit ist in der Ukraine oft erfreulich einfacher als in Russland. Seit der ukrainischen Unabhängigkeit sind eine Reihe von Quellenbeständen erstmals zugänglich gemacht worden, die es in der Zukunft ermöglichen werden, die Geschichte des Zweiten Weltkriegs in der Ukraine noch besser zu erforschen. ${ }^{2}$

Die Bedeutung des „Großen Vaterländischen Krieges“ für die Ukrainer sahen westliche und ukrainische Forscher zunächst vor allem in der Vereinigung aller Ukrainer in einem ukrainischen Staat - der Ukrainischen Sozialistischen Sowjetrepublik - sowie in den enormen Menschenverlusten und Besatzungsschäden. In der ukrainischen Geschichtswissenschaft erfolgte nach dem Zusammenbruch der Sowjetunion eine Umdeutung der Sowjethistoriographie unter nationalen Vorzeichen und ukrainische Historiker richteten ihr Augenmerk insbesondere auf die Rolle der Organisation ukrainischer Nationalisten (OUN) und der Ukrainischen Aufstandsarmee (UPA), die die Bevölkerung für einen unabhängigen ukrainischen Nationalstaat zu mobilisieren versuchten. Dies ist zwar aufgrund der Erfahrungen der Sowjetzeit verständlich, fördert aber im Hinblick auf die Geschichte der Ukraine im Zweiten Weltkrieg auch wieder nur ein verzerrtes Bild zutage, zumal die ukrainische Nationalbewegung in den verschiedenen ukrainischen Regionen nicht gleichermaßen von Bedeutung war sondern sich vor allem auf die westukrainischen Gebiete konzentrierte. Für die Mehrheit der Bevölkerung in der Zentral- und Ostukraine spielte die ,nationale Fra-

\footnotetext{
1 Vgl. die exzellente Überblicksdarstellung mit zahlreichen Literaturhinweisen von DiETER POHL Schlachtfeld zweier totalitärer Diktaturen - die Ukraine im Zweiten Weltkrieg, in: Österreichische Osthefte 42 (2000) 3/4, S. 339-362.

2 Vgl. TAnja Penter Collaboration on Trial: New Source Material on Soviet Postwar Trials Against Collaborators, in: Slavic Review 64 (2005), S. 780-790.
} 
ge" in den Kriegsjahren hingegen eine untergeordnete Rolle, wie Regionalstudien gezeigt haben. ${ }^{3}$

Die Rolle der OUN- und UPA-Aktivisten wird in der Forschung und in der Öffentlichkeit bis heute kontrovers diskutiert. Mehrere parlamentarische und historische Untersuchungskommissionen widmeten sich dieser Frage. ${ }^{4}$ Problematisch ist vor allem die Beteiligung von OUN- und UPA-Mitgliedern an Pogromen gegen die jüdische Bevölkerung und an deutschen Massenverbrechen. Zudem betrieben Teile der UPA ab 1943 in Wolhynien und Ostgalizien eine Politik der ,ethnischen Säuberungen" gegen die polnische Minderheit und gegen die Juden. ${ }^{5}$ In der Westukraine werden die Mitglieder der OUN und UPA und sogar Mitglieder der SS-Division Galizien bis heute als Freiheitskämpfer und Helden verherrlicht. Dagegen ist die ukrainische Beteiligung an Kriegsverbrechen bisher erst in Ansätzen erforscht worden und stellt bis heute auch in der ukrainischen Diaspora einen blank spot in der kollektiven Erinnerung dar, wie John-Paul Himka konstatierte. Himka kritisiert insbesondere, dass in der ukrainischen Diaspora bis heute unterschiedliche Standards bei der Bewertung von Kriegsverbrechen, die von Ukrainern begangen wurden, im Gegensatz zu denen, die an Ukrainern verübt wurden, vorherrschen. Häufig gehe der ukrainische Opfer-Diskurs einher mit einem Leugnen der Verantwortung für begangene Kriegsverbrechen. ${ }^{6}$

Zunehmend öffnet sich die ukrainische Forschung in den letzten Jahren aber auch für diese in der Sowjethistoriographie lange tabuisierten Aspekte der Geschichte des Zweiten Weltkriegs, wie den Besatzungsalltag, den Holocaust oder die einheimische Kollaboration. In drei Zentren zur Erforschung der Geschichte des Holocaust in Kiev, Charkiv und Dnipropetrovs'k wird die Ermordung der ukrainischen Juden zunehmend auch von nicht-jüdischen Historikern untersucht. Zugleich erfuhr die Wahrnehmung der Opfer des Nationalsozialismus in den letzten Jahren eine beständige Erweiterung, so dass auch ehemalige Zwangsarbeiter und Kriegsgefangene sowie Roma erstmals in den Fokus der Historiker rückten. Deutsche Entschädigungs- und Begegnungsprogramme wirkten hier zum Teil als Motor für geschichtspolitische Debatten und die öffentliche Anerkennung neuer Opfergruppen. Endlich

Vgl. KAREL C. Berkhoff Harvest of Despair. Life and Death in Ukraine under Nazi Rule. Cambridge, Mass., London 2004, S. 205-231; HIROAKI KUROMIYA Freedom and Terror in the Donbas. A Ukrainian-Russian Borderland, 1870s - 1990s. Cambridge 1998, S. 277-282; TANJA PENTER Kohle für Stalin und Hitler. Arbeiten und Leben im Donbass 1929 bis 1953. Essen 2010.

4 Vgl. Orhanizacija ukrajins'kych nacionalistiv i Ukrajins'ka povstans'ka armija. Istoryčne narysy. Ky jiv 2005; Protystojannja. Zverennja, zajavy, lysty hromads'kych orhanizacij, polityčnych partij, hro madjan Ukrajiny do Komisiji z vyvčennja dijal'nosti OUN-UPA. 1996-1998rr. Kyjiv 1999; WiLfried JiLge The Politics of History and the Second World War in Post-Communist Ukraine (1986/1991 - 2004/ 2005), in: Jahrbücher für Geschichte Osteuropas 54 (2006), S. 50-81.

5 Vgl. dazu Grzegorz MOTYKA Der polnisch-ukrainische Gegensatz in Wolhynien und Ostgalizien, in: Bernhard Chiari (Hg.) Die polnische Heimatarmee. Geschichte und Mythos der Armia Krajowa seit dem Zweiten Weltkrieg. München 2003, S. 531-548.

6 Vgl. JohN-Paul Hrmka War Criminality: A Blank Spot in the Collective Memory of the Ukrainian Diaspora, auf der Homepage der Universität Wien: www.univie.ac.at/spacesofidentity/ _Vol_5_1/_HTML/Himka.html, (Zugriff 24.4.2010). 
- im Grunde Jahrzehnte zu spät - begann man in mehreren Oral history-Projekten die erzählten Erinnerungen der Kriegsgeneration zu sammeln und aufzuzeichnen.?

Der Krieg und die deutsche Besatzung wurden von den Bevölkerungen in den verschiedenen Regionen der Ukraine - vor allem in den altsowjetischen Gebieten der Zentral- und Ostukraine sowie in den 1939 erst im Zuge des Hitler-Stalin-Paktes annektierten westukrainischen Gebieten - sehr unterschiedlich erfahren. Dies äußert sich bis heute in regional heterogenen Erinnerungskulturen, in denen sich sowjetische und ukrainisch-nationale Symbole und Traditionen auf komplizierte Art und Weise überlagern. ${ }^{8}$ Während in der Zentral- und Ostukraine die Erinnerung an den Sieg im „Großen Vaterländischen Krieg“ dominiert und die Rote Armee als Befreier gesehen wird, deuten viele Westukrainer den Krieg primär als Kampf der OUN- und UPA-Aktivisten gegen die sowjetische Besatzung. Es existiert also bis heute eine Vielzahl von unterschiedlichen Narrativen zur Geschichte der Ukraine im Zweiten Weltkrieg, aus denen man den Eindruck gewinnen könnte, dass es sich um völlig verschiedene Ereignisse gehandelt habe.

Ich möchte an dieser Stelle daher für stärker integrierende Forschungsperspektiven plädieren, die sich bemühen, im Gegensatz zu der bisher verbreiteten singulären Betrachtung einzelner Phänomene, das breite Spektrum unterschiedlicher Kriegserfahrungen der Ukrainer (und der in der Ukraine lebenden nicht-ukrainischen Minderheiten) analytisch zusammenzuführen. Erstens impliziert dies, die Bedeutung des Zweiten Weltkriegs für die Ukrainer nicht primär aus der Perspektive der Nationsund Staatsbildung zu betrachten, sondern auch andere Aspekte der Kriegs- und Besatzungserfahrung, die den Großteil der Bevölkerung der Ukraine gleichermaßen betrafen, wie der Arbeitseinsatz für die Besatzer oder die Zwangsdeportationen ins Deutsche Reich, stärker in den Blick zu nehmen und damit die Nationalismusforschung mehr in die Sozial- und Alltagsgeschichte zu integrieren. Dies bedeutet nicht, dass diese nationale Perspektive, die ja andernorts in diesem Band am Beispiel der OUN und UPA behandelt wird, ${ }^{9}$ unwichtig wäre. Vielmehr geht es darum, die Perspektive auf weitere Aspekte der Geschichte der Ukraine im Zweiten Weltkrieg zu erweitern, die in ihrer Bedeutung auch für die Entwicklung der ukrainischen Nation in der Forschung bisher unterbelichtet sind.

Vgl. Alexander von Plato, Almut Leh, Christoph Thonfeld (Hg.) Hitlers Sklaven. Lebens geschichtliche Analysen zur Zwangsarbeit im internationalen Vergleich. Wien, Köln, Weimar 2008; G.H. HRINČENKO Nevyhadane usni istoriji ostarbajteriv. Charkiv 2004; BORYS ZABARKO „Nur wir haben überlebt": Holocauist in der Ukraine. Zeugnisse und Dokumente. Berlin 2004; PATRICK DEsBois Der vergessene Holocaust: Die Ermordung der ukrainischen Juden. Eine Spurensuche. Berlin 2009. Die Ergebnisse eines weiteren Interviewprojektes von Dmytro Tytarenko und Tanja Penter zu den Besatzungs- und Nachkriegserfahrungen der Bevölkerung im Donbass sollen in Kürze (in russischer Sprache) veröffentlicht werden. Zudem führt John-Paul Himka gerade gemeinsam mit seiner Tochter ein Interviewprojekt unter Aktivisten der OUN und UPA durch.

8 Vgl. dazu den von STEFAN TROEBST und WILFRIFD JiLGE herausgegebenen Band: Divided Historical Cultures? World War II and Historical Memory in Soviet and post-Soviet Ukraine, in: Jahrbücher für Geschichte Osteuropas 54 (2006), H. 1.

? Vgl. den Beitrag von FranK GOLCZEWSKI in diesem Band. 
Zweitens erscheint es sinnvoll, bei der Untersuchung der Kriegs- und Besatzungszeit auch die unterschiedlichen Vorkriegserfahrungen der Bevölkerung stärker zu berücksichtigen und auf diesem Wege die bisher noch weitgehend losgelöst voneinander stehenden Forschungsfelder zum Nationalsozialismus und zum Stalinismus miteinander zu verknüpfen bzw. die Erfahrungen der Ukrainer in Polen, Rumänien und der Tschechoslowakei in der Zwischenkriegszeit stärker einzubeziehen. ${ }^{10}$

Drittens scheint die bisher in der Geschichtswissenschaft dominante separate Betrachtung von Nationalismus, Kollaboration, Anpassung oder Widerstand den komplexen Kriegsbiographien der Menschen nicht angemessen zu sein, die sich vielmehr durch eine starke Vermischung und zum Teil auch Gleichzeitigkeit all dieser Phänomene auszeichneten. Die von der Historiographie vorgenommenen Trennlinien decken sich also nicht mit den realen Erfahrungswelten der Kriegsgeneration. Alte Stereotype, die Ukrainer mit Nationalisten und Antisemiten gleichsetzen und sich bis heute hartnäckig in der westeuropäischen Öffentlichkeit halten, können durch eine solche Forschungsperspektive möglicherweise revidiert werden.

Viertens und letztens scheint mir eine stärker integrierende Perspektive auch im Hinblick auf die deutschen Besatzungsverbrechen geboten. Die bisher vorherrschende singuläre Betrachtung des Holocaust, die auf der zentralen Stellung des Holocaust in der westeuropäischen Ethik und Erinnerungskultur basiert, würde dann durch eine Sichtweise ersetzt, die den Holocaust stärker in den Kontext anderer Besatzungsverbrechen, wie der Ermordung der sowjetischen Kriegsgefangenen, der verbrannten Dörfer, ethnischer Säuberungen oder der Zwangsarbeit, einbettet und integriert. Die von deutscher Seite vorangetriebene Hervorhebung des jüdischen Leids, beispielsweise im Zusammenhang mit Wiedergutmachungsprogrammen für NS-Opfer, stößt in der ukrainischen ebenso wie in anderen postsowjetischen Gesellschaften häufig auf Ablehnung und fördert so die Konkurrenz zwischen jüdischen und nicht-jüdischen Opfern. Dies erklärt sich zum Teil aus der jahrzehntelangen Unterdrückung der jüdischen Erinnerung an den Holocaust in der Sowjetunion sowie aus bis heute in der Ukraine existierenden antisemitischen Tendenzen. Andererseits basiert diese Haltung aber auch auf der Tatsache, dass in den besetzten sowjetischen Gebieten sehr viele nicht-jüdische Opfer aus der Zivilbevölkerung oder unter den sowjetischen Kriegsgefangenen den deutschen Massenverbrechen zum Opfer fielen. Die Bevölkerung erfuhr die deutschen Verbrechen als Gesamtkontext, wie in Interviews mit Zeitzeugen immer wieder deutlich wird, und deshalb stoßen die singulären Forschungsansätze der Historiker bei den Zeitzeugen häufig auf Unverständnis. Im Folgenden werde ich zwei der genannten Perspektiven etwas näher ausführen.

10 Ich habe versucht, diesen Ansatz für den ostukrainischen Donbass aufzugreifen: Vgl. PENTER Kohle für Stalin und Hitler. 


\section{Ukrainische Nation und Zwangsarbeitserfahrungen}

Ein bedeutender Teil der Bevölkerung der Ukraine machte in den Kriegsjahren spezifische Arbeitserfahrungen unter deutscher Herrschaft, deren Bedeutung auch für die ukrainische Nachkriegsentwicklung kaum zu unterschätzen ist, von der Forschung bisher aber erst in Ansätzen zur Kenntnis genommen wurde. Die deutsche Besatzungspolitik, die primär auf die wirtschaftliche Ausbeutung der Ukraine zielte, der dabei die Rolle einer Kolonie zugedacht war, schloss auch die Ausbeutung der Arbeitskraft der einheimischen Bevölkerung von Anfang an ein. Im August 1941 hatte der Reichsminister für die besetzten Ostgebiete eine allgemeine Arbeitspflicht für alle Einwohner der Ostgebiete im Alter zwischen 18 und 45 Jahren sowie einen Arbeitszwang für Juden im Alter von 14 bis 60 Jahren unter Androhung von massiven Strafen bei Nichteinhaltung eingeführt. ${ }^{11}$ Die Arbeitseinsatzpolitik bildete ein zentrales Element der Besatzungspolitik, das den Alltag der Mehrheit der ukrainischen Bevölkerung maßgeblich prägte. Über die quantitative Dimension dieses Arbeitseinsatzes lassen sich anhand der verfügbaren Quellen allerdings bisher nur ungefähre und punktuelle Aussagen treffen. Im Juli 1943 standen 7,5 (44\%) der 16,9 Millionen Menschen im Reichskommissariat Ukraine im Arbeitseinsatz; weitere 3,2 (46\%) der etwa 7 Millionen zählenden Bevölkerung kamen in den unter Militärverwaltung stehenden ostukrainischen Gebieten hinzu. Über vier Fünftel der Beschäftigten arbeiteten in der Landwirtschaft. Laut einer groben Statistik der Chefgruppe Arbeit des Wirtschaftsstab Ost arbeiteten Ende 1942 20,8 Millionen Menschen in den besetzten Ostgebieten in der Landwirtschaft, aber nur 1,1 Millionen in der Industrie. Hinzu kamen weitere Millionen Arbeitskräfte, die beim Bau von Eisenbahnverbindungen, Straßen, Flugplätzen, Brücken und Befestigungsanlagen beschäftigt waren sowie Hunderttausende, die im Gefolge der Wehrmacht eingesetzt waren. ${ }^{12}$

Zudem waren zahlreiche Ukrainer als Zwangsarbeiter ins Reich deportiert worden. Im Ganzen wurden aus der Ukraine etwa 2,14 der insgesamt etwa 3,1 Mio. sogenannten „Ostarbeiter" nach Deutschland verschleppt. ${ }^{13}$ Hinzu kamen Hunderttausende sowjetischer Kriegsgefangener ukrainischer Nationalität. ${ }^{14}$ In den Gebieten un-

11 Vgl. dazu ausführlich Tanja Penter Arbeiten für den Feind in der Heimat. Der Arbeitseinsatz in der besetzten Ukraine 1941-1944, in: Jahrbuch für Wirtschaftsgeschichte 1 (2004), S. 65-94; DiES., Zwangsarbeit - Arbeit für den Feind. Der Donbass unter deutscher Okkupation, 1941-1943, in: Geschichte und Gesellschaft 31 (2005), S. 68-100.

12 Vgl. Statistik der Chefgruppe Arbeit des Wirtschaftsstabes Ost zum Arbeitseinsatz in den bis Ende 1942 besetzten Ostgebieten, in: RGVA (Russländisches Staatliches Kriegsarchiv), F. 700, Op. 1, D. 82, S. 1-2 sowie ROLF-DiETER MüLLER Die deutsche Wirtschaftspolitik in den besetzten sowjetischen Gebieten 1941-1943. Der Abschlußbericht des Wirtschaftsstabes Ost und Aufzeichnungen eines Angehörigen des Wirtschaftskommandos Kiew. Boppard am Rhein 1991, S. 477.

13 Etwa 55\% der 3,1 Mio. waren ethnische Ukrainer. Vgl. PENTER Arbeiten für den Feind sowie MARK SPOERER Zwangsarbeit unter dem Hakenkreuz. Ausländische Zivilarbeiter, Kriegsgefangene und Häftlinge im Deutschen Reich und im besetzten Europa 1939-1945. München 2001, S. 80.

14 Vgl. Pavel Poljan Deportiert nach Hause. Sowjetische Kriegsgefangene im „Dritten Reich“ und ihre Repatriierung. München 2001, S. 165. 
ter Militärverwaltung waren die Arbeitskräftemobilisierungen für den Reichseinsatz, gemessen an der jeweiligen Bevölkerungszahl, deutlich größer als im zivilverwalteten Reichskommissariat Ukraine - was die noch immer verbreitete Auffassung von der „milderen" Besatzungsherrschaft unter deutscher Militärverwaltung relativiert. Die Forschung hat zwar bereits vor längerer Zeit festgestellt, dass das Leben in den Gebieten unter Militärverwaltung für die Bevölkerung kaum besser war als im Reichskommissariat Ukraine. Sie hat dieses aber bisher nahezu ausschließlich unter dem Aspekt der Massenvernichtung und Verbrechen gegen einzelne Bevölkerungsgruppen untersucht. Der Arbeitseinsatz war hingegen ein Bereich, der den Großteil der lokalen Bevölkerung direkt betraf. Und auch dort erwies sich die Militärverwaltung für die lokale Bevölkerung somit nicht als vorteilhafter.

Bei der Rekrutierung der Arbeitskräfte setzten die Besatzungsbehörden - wenn erforderlich - von Beginn an Zwangsmaßnahmen und physische Gewalt ein, wobei den einheimischen Polizeikräften eine zentrale Rolle zukam. Allerdings musste nicht in jedem Fall Zwang angewandt werden, denn es gab sowohl für den Reichseinsatz als auch für den Arbeitseinsatz vor Ort auch freiwillige Meldungen. Dabei ist festzustellen, dass der Reichseinsatz umso mehr von seiner anfänglichen "Attraktivität" verlor, desto mehr über die wirklichen Lebensbedingungen der „Ostarbeiter“ im Reich bekannt wurde und je mehr sich die Ernährungslage der lokalen Arbeitskräfte vor Ort besserte. Darüber hinaus hing die ,Arbeitswilligkeit“ der Bevölkerung aber auch von den Ereignissen an der Front ab. Insbesondere in den frontnahen Gebieten konnte die Furcht vor einer Rückkehr der Roten Armee und vor Vergeltungsaktionen gegen alle, die für die Besatzer gearbeitet hatten, auch zu Arbeitsverweigerungen führen.

Die konkreten Bedingungen des Arbeitseinsatzes waren in den besetzten Gebieten - ebenso wie beim Reichseinsatz - für die Sowjetbürger durchaus unterschiedlich und von vielfältigen Faktoren abhängig. Auf dem Land waren die Arbeits- und Lebensbedingungen, insbesondere der Zugang zu Nahrungsmitteln, vielfach besser als in der Stadt. Die Besatzungsbehörden waren sich bewusst, dass für den Erfolg der landwirtschaftlichen Produktion vor allem der gute Wille der Bevölkerung zur Mitarbeit entscheidend war, weil ,man nicht hinter jedem Pflug einen Polizisten laufen lassen" konnte und es an deutschen Aufsichtskräften mangelte. ${ }^{15}$ Wie Karel Berkhoff gezeigt hat, verfügte der Großteil der ukrainischen Bauern unter der Besatzung im Durchschnitt - mit regionalen und lokalen Unterschieden - über mehr Lebensmittel als in der sowjetischen Vorkriegszeit, was vor allem auf ihre extreme Armut unter der Sowjetherrschaft hinweist. ${ }^{16}$

Der Arbeitseinsatz von Frauen gewann unter den Deutschen noch weiter an Bedeutung, wobei die Deutschen an die sowjetische Vorkriegsentwicklung anknüpften. Frauen stellten nicht nur über die Hälfte der deportierten „Ostarbeiter", sondern

15 Vgl. Programm für die Ostbesprechung unter Vorsitz des Reichsmarschalls (undatiert), in: RGVA, F. 700, Op. 1, D. 49, S. 64 sowie Vermerk der Geschäftsgruppe Ernährung beim Beauftragten für den Vierjahresplan vom 27. November 1942, in: Ebda., S. 69-70.

16 Vgl. BERKHOFF Harvest of Despair, S. 93-143. 
wurden auch vor Ort vermehrt als Arbeitskräfte eingesetzt. In den Gebieten unter Militärverwaltung waren im Juli 194360 Prozent aller Beschäftigten Frauen. ${ }^{17}$

Die ukrainischen Arbeitskräfte machten während ihrer Zwangsarbeit vielfältige kulturelle Erfahrungen mit den Deutschen, wobei im direkten Kontakt verschiedene Selbst- und Fremdbilder aufeinanderprallten und ältere stereotype Wahrnehmungen verfestigt oder aufgebrochen wurden. Neben dem allgegenwärtigen Terror manifestierten sich in den Arbeitsbeziehungen auch Elemente von Normalität. Die Arbeit für den Feind bedeutete manchmal auch Schutz und konnte für einige Rettung vor der Vernichtung oder vor der Deportation ins Reich bedeuten. Die zentrale Bedeutung dieser Erfahrungen zeigt sich bereits daran, dass Überlebende sich bis heute (z.B. bei Interviews) sehr detailliert an ihre (vergleichsweise kurzen) Erfahrungen unter deutscher Besatzung oder als „Ostarbeiter“ im Deutschen Reich erinnern. Für viele blieb dies bis zum Ende der Sowjetunion der einzige nähere Kontakt zu westlichen Ausländern bzw. die einzige „Reise“ in den Westen. Die zentrale Bedeutung des „Produktionsfaktors Mensch“ für die deutsche Kriegswirtschaft und der große wirtschaftliche Nutzen des Arbeitseinsatzes von Sowjetbürgern für das Reich sind in der Forschung heute unbestritten. Noch kaum erforscht ist hingegen die Frage nach den Rückwirkungen dieses Arbeitseinsatzes auf die ukrainische Nachkriegsgesellschaft, die - ganz abgesehen von den wirtschaftlichen Schäden - beträchtlich zu sein scheinen. Diejenigen, die für den Feind gearbeitet hatten, standen nach dem Krieg unter dem Pauschalverdacht des „Vaterlandsverrats“. Insbesondere die heimkehrenden „Ostarbeiter" und Kriegsgefangenen bekamen dies nach ihrer Repatriierung in Form von Repressalien und Diskriminierungen zu spüren. Zahlreiche Sowjetbürger, die in leitender Funktion mit den Deutschen zusammengearbeitet hatten, wurden von sowjetischen Militärtribunalen als „Kollaborateure“ verurteilt. Viele ehemalige Zwangsarbeiter mussten nach ihrer Heimkehr in die Sowjetunion dort erneut Zwangsarbeit beim Wiederaufbau leisten, beispielsweise in den Kohlenbergwerken des Donbass. Sie erfuhren Stigmatisierungen und berufliche Diskriminierungen, die bis zum Ende der Sowjetzeit andauerten. Die Heimkehrer wurden somit Opfer zweier Diktaturen. Unklarheit herrscht jedoch bis heute über die genaue Art und das Ausmaß der Repressionen gegen die Repatriierten. Der Blick auf die Biographien ehemaliger Zwangsarbeiter fördert kein eindeutiges Bild zutage. So findet man unter ihnen sowohl solche, die ihr Leben lang in ihren beruflichen Karrierechancen stark eingeschränkt waren, als auch solche, die es dennoch zu Universitätsprofessoren oder in Belarus' in einem Fall sogar zum Verteidigungsminister brachten. Welchen Gesetzmäßigkeiten das Leben nach der Repatriierung folgte und welche Faktoren für das weitere Fortkommen des Einzelnen entscheidend waren, werden entsprechende Mikrostudien erst in der Zukunft ans Licht bringen: Beispielsweise die Studie der Kiever Historikerin Tatjana Pastušenko, die den weiteren Werdegang der Repatrïerten in der Stadt Kiev untersucht.

17 Vgl. Ul.rich Herbert Fremdarbeiter. Politik und Praxis des „Ausländer-Einsatzes“ in der Kriegswirtschaft des Dritten Reiches. Berlin, Bonn 1985, S. 272 sowie MÜLter Die deutsche Wirtschaftspolitik, S. 304. 
Die Diskriminierungen und Stigmatisierungen betrafen nicht nur die aus dem Reich repatriierten „Ostarbeiter" und Kriegsgefangenen, sondern auch mehrere Millionen Einwohner der besetzten Gebiete, bei denen ab 1943 ein diesbezüglicher Vermerk im Ausweis vorgenommen wurde. Dieser Vermerk konnte Karrierechancen unter Umständen einschränken oder andere Probleme mit sich bringen. Jemand, der in den obligatorischen Fragebögen angab, dass er im besetzten Gebiet gelebt hatte, besaß kaum eine Chance, von einer höheren Bildungseinrichtung zum Studium angenommen zu werden oder auf anderem Wege in eine berufliche Leitungsposition zu gelangen. Valentina Ivanovna aus Donec'k, die die deutsche Besatzung als 14-jährige miterlebte, erinnert sich an die Nachkriegsjahre: „Die Diskriminierung zeigte sich nicht so offen. Das war mehr unterschwellig. Aber der Mensch spürte, dass er kein vollwertiges Mitglied des Kollektivs war. An meinem Arbeitsplatz als Buchhalterin habe ich immer gespürt, dass die Haltung mir gegenüber nicht so war wie gegenüber den Evakuierten." 18

Was bedeuteten diese NS-Zwangsarbeiter für die ukrainische Nachkriegsentwicklung? Was bedeutete es für die Ukraine, dass Millionen von Menschen einen Blick hinter den ,Eisernen Vorhang“ getan hatten und aufgrund ihrer Erfahrungen im Westen oder im besetzten Gebiet möglicherweise eine gewisse Resistenz gegenüber den Mythen der Sowjetpropaganda entwickelt hatten? Die Reintegration der Repatriierten und der Bevölkerung im besetzten Gebiet in die Sowjetgesellschaft stellte für das stalinistische Regime in der Nachkriegszeit eine immense Herausforderung dar. Wie verliefen die kollektiven Biographien der ehemaligen Zwangsarbeiter nun in der Sowjetunion weiter? Welche Möglichkeiten hatten sie, ihre Traumatisierungen aus der Kriegszeit aufzuarbeiten? Wie wurden sie in der öffentlichen Erinnerungskultur repräsentiert? Es gehört zu den Besonderheiten der sowjetischen Entwicklung, dass bis zum Ende der Sowjetunion keine größere öffentliche Debatte über das Thema NS-Zwangsarbeit stattgefunden hat und dass die Anfänge einer Debatte in der ausgehenden Sowjetunion maßgeblich durch deutsche Entschädigungsdiskussionen angestoßen wurden.

Die Erfahrung der Zwangsarbeit unter deutscher Herrschaft war eine gemeinsame Erfahrung, die Ukrainer in den heterogenen Regionen der Ost-, Zentral- und Westukraine gleichermaßen teilten. Die ehemaligen ukrainischen Zwangsarbeiter haben erst in jüngster Zeit damit begonnen, ihre Erinnerung an die NS-Zwangsarbeit öffentlich zu verarbeiten, beispielsweise in Formen des ritualisierten öffentlichen Erzählens oder auch bei Theateraufführungen, die sich in Kiev heute großer Beliebtheit erfreuen. Die Zwangsarbeitserfahrung bildet einen Anknüpfungspunkt für eine über die regionalen und ethnischen Trennlinien hinausreichende Erinnerung an den Zweiten Weltkrieg.

Es bleibt ein weiteres, besonders dunkles und bisher kaum untersuchtes Kapitel der Besatzungsgeschichte zu erwähnen: Der Arbeitseinsatz sowjetischer Kriegsgefangener, die am unteren Ende der Arbeitskräfte-Hierarchie standen. Während

18 Vgl. Interview mit Valentina Ivanovna (geb. 1927) aufgezeichnet im Dezember 2003 in Donec'k. 
zum Arbeitseinsatz der sowjetischen Kriegsgefangenen im Deutschen Reich seit Ende der 1960er Jahre mehrere umfassende Untersuchungen erschienen sind und seit Anfang der 1980er Jahre zudem zahlreiche Arbeiten zu einzelnen Kriegsgefangenenlagern vorliegen, wissen wir über die Situation der sowjetischen Kriegsgefangenen in den besetzten Gebieten nur sehr wenig. ${ }^{19}$ Die Ukraine war einer der wichtigsten Schauplätze des Massensterbens von Kriegsgefangenen, das sich vor den Augen der lokalen Bevölkerung ereignete. ${ }^{20}$ Tausende kamen bereits auf den langen Märschen in die Lager um. Die Masse der Kriegsgefangenen starb seit Oktober 1941 in den östlichen Durchgangslagern und vor allem in den großen Stammlagern im Reichskommissariat Ukraine - ein großer Teil von ihnen verhungerte. ${ }^{21} \mathrm{Im}$ ostukrainischen Steinkohlenrevier des Donbass wurden Zehntausende von Kriegsgefangenen als Arbeitskräfte im Bergbau eingesetzt. Ihre Sterblichkeit betrug in den BergbauStammlagern zeitweilig über zwölf Prozent monatlich. In vielen Lagern herrschten Fleckfieber und Typhus. ${ }^{22}$ Nach sowjetischen Angaben kamen allein im Gebiet Stalino (Donec'k) im Besatzungszeitraum bis zu 150.000 sowjetische Kriegsgefangene ums Leben. ${ }^{23}$ Die Arbeitsleistung der Kriegsgefangenen war gering, was neben ihrem schlechten Gesundheitszustand auch daran lag, dass der größte Teil von ihnen Bauern waren und nur sehr wenige Bergleute oder andere Facharbeiter. Obwohl die Kriegsgefangenen die Bergbauunternehmen nur etwa halb so viel kosteten wie die Zivilarbeiter, konnte ihr Einsatz daher nicht im wirtschaftlichen Interesse des Unternehmens liegen.

In den Erinnerungen der Zeitzeugen häufen sich Berichte über die Solidarität und Hilfeleistungen der lokalen Bevölkerung gegenüber den Kriegsgefangenen, die jedoch immer wieder von den Deutschen brutal unterbunden worden seien. Dieser Topos, der gewisse Parallelen zu dem in der deutschen Nachkriegsgesellschaft verbreiteten Narrativ, wonach die deutsche Bevölkerung den Zwangsarbeitern heimlich

19 Vgl. dazu mit zahlreichén Literaturhinweisen PENTER Arbeiten für den Feind. Einen ersten Überblick zum Arbeitseinsatz der Kriegsgefangenen in der Ukraine liefert O. POTYL'ČAK Trudovi resursy radjans'kych vijs'kovopolonenych ta "ostarbajteriv“ z Ukrajiny u nacists'kij vijs'kovij ekonomici v roky druhoji svitovoji vijny. Kyjiv 1998.

20 Nach der jüngsten und bisher umfassendsten Zusammenstellung über Lager, Gefängnisse und Ghettos in der Ukraine existierten dort insgesamt 242 Kriegsgefangenenlager. Vgl. Handbuch der Lager, Gefängnisse und Ghettos auf dem besetzten Territorium der Ukraine (1941-1944), in Dovidnyk pro tabory, tjurmy ta hetto na okupovanij terytoriji Ukrajiny (1941-1944). Kyjiv/Kiev 2000. Der Band ist zweisprachig deutsch-ukrainisch.

21 Vgl. PoHL Schlachtfeld zweier Diktaturen, S. 349-350. Am Jahresende 1941 starben in den Lagern im RKU täglich etwa 2.500 Gefangene. Vgl. Christian STREIT Keine Kameraden. Die Wehrmacht und die sowjetischen Kriegsgefangenen 1941-1945. 4. Aufl., Bonn 1997, S. 133.

22 Vgl. dazu ausführlich TANJA Penter Zwischen Hunger, Terror und einer ,glücklichen Zukunft“. Der Arbeitseinsatz im Steinkohlenbergbau des Donezbeckens unter deutscher Besatzung 1941 bis 1943, in: Klaus TenFelde, Hans-Christoph SEIDEL (Hg.) Zwangsarbeit im Bergwerk. Bd. 1: Forschungen, Essen 2005, S. 433-466.

23 O. Ionov Zločynstva Nimciv u Donbasi. Kyjiv 1946, S. 15. Vgl. zur Behandlung der sowjetischen Kriegsgefangenen in der Ukraine auch KAREL C. BERKHOFF The „Russian“ Prisoners of War in Nazi-Ruled Ukraine as Victims of Genocidal Massacre, in: Holocaust and Genocide Studies 15 (2001), S. 1-32. 
Butterbrote zugesteckt habe, aufweist, diente (in beiden Fällen) möglicherweise auch dazu, unterschwellige Schuldgefühle zu überdecken. Die größere oder geringere Solidarität der lokalen Bevölkerung mit den jeweiligen verfolgten Gruppen hatte (wenngleich begrenzte) Auswirkungen auf deren Überlebenschancen im besetzten Gebiet und verdient in der Zukunft in der Forschung noch größere Beachtung. Durch die größere Hilfsbereitschaft der ukrainischen Bevölkerung gegenüber den Roma erklären sich Historiker beispielsweise in jüngster Zeit die Tatsache, dass in der besetzten Ukraine ein bedeutender Teil der Roma überleben konnte, während ukrainische Juden die deutsche Vernichtungspolitik nur vereinzelt überlebten. ${ }^{24}$

\section{Die Komplexität der Kriegsbiographien}

Jahrzehntelang herrschte in der Forschung ein Bild vor, das letztlich die schablonenhaften Schwarz-Weiß-Zeichnungen der Sowjethistoriographie übernahm, die sowjetische Bevölkerung entweder in Nationalisten, Kollaborateure, Widerstandskämpfer oder Opfer unterteilte und die Phänomene jeweils in separaten Einzelstudien untersuchte. Dieses Bild gerät nun durch die stärkere Wahrnehmung der individuellen Kriegsbiographien der Menschen zunehmend ins Wanken. Die Loyalitäten im Krieg wandelten sich laufend und viele Kriegsbiographien zeichneten sich durch die Gleichzeitigkeit des Ungleichzeitigen und die Vermischung von Gegensätzen aus. Die scharfen Trennlinien, die Historiker im Nachhinein schufen, existierten im realen Alltag des Krieges nicht. Ich möchte dies im Folgenden an einigen Beispielen ausführen.

Bei der Betrachtung der Kriegsbiographien von in der Nachkriegs-Ukraine verurteilten Kollaborateuren ${ }^{25}$ ließen sich zahlreiche stereotype Vorstellungen revidieren. ${ }^{26}$ Es waren nicht nur ukrainische Nationalisten, die als Hilfspolizisten, Mitglieder der lokalen Verwaltungen oder leitende Arbeitskräfte der Wirtschaftsbetriebe mit den Deutschen zusammenarbeiteten, sondern ebenso Russen und andere in der Ukraine lebende ethnische Minderheiten. Im ostukrainischen Donezbecken dienten - bedingt durch die dortige ethnische Bevölkerungsstruktur - offenbar nicht wenige Russen in den Reihen der „ukrainischen Hilfspolizei“, ebenso Ukraine-Deutsche und andere Minderheiten. Es stimmt auch nur zum Teil, dass sich die Kollaborateure aus Perso-

24 Vgl. dazu den Themenband von Holokost i sučasnist‘ 2 (6) (2009).

25 Von der ukrainischen Geschichtsschreibung ist die Kollaboration von Bevölkerungsgruppen mit den Besatzern bisher noch kaum untersucht worden und wird gerne als Randphänomen abgetan. Erste Ansätze bei A. SKOROBOHATOv Charkivs'ke Mis'ke Samovrjaduvannja v 1941-1943 rokach, in: Berezil' 7-8 (1996), S. 139-162; H. 9-10 (1996), S. 145-164; Ders. Charkiv u časi nimec’koji okupaciji (1941-1943). Charkiv 2004. Vgl. zudem die jüngst erschienene erste Monographie zur Kollaboration in der Ukraine von V.O. SAJKAN Kolaboracionizm na terytoriji Rejchskomisariatu „Ukrajina" i vijs'kovoji zony v roky Druhoji svitovoji vijny. Kryvyj Rih 2005.

26 Vgl. dazu ausführlich TANJA PENTER Local Collaborators on Trial. Soviet War Crimes Trials Under Stalin (1943-1953), in: Cahiers du Monde russe 49 (2008), 2-3, S. 341-364, sowie DiEs. Collaboration on Trial. 
nengruppen rekrutierten, die der Sowjetmacht feindlich gegenüber standen, weil sie unter ihr Repressionen erlitten hatten. Nicht selten fanden sich in Leitungspositionen der einheimischen Verwaltungsorgane auch langjährige Mitglieder der Kommunistischen Partei, die bereits vor dem Krieg unter der Sowjetherrschaft verantwortungsvolle Positionen im Sowjetapparat bekleidet hatten. ${ }^{27}$ So stellte die Parteiorganisation im ostukrainischen Stalino (heute: Donec'k) nach der Befreiung des Gebietes fest, dass in einigen Bezirken eine beachtliche Zahl von Kommunisten im besetzten Gebiet verblieben war, und konstatierte: „Ein Teil der Kommunisten erwies sich als Feiglinge, ein anderer als Ballast, ein weiterer als Betrüger und Vaterlandsverräter." ${ }^{28}$ Auch im Gebiet Vorošilovgrad waren nach Angaben des NKVD von 450 Personen, die kurz nach der Befreiung als „Verräter" festgenommen worden waren, die wenigsten unter der Sowjetmacht verfolgt worden, während die Mehrzahl auf den ersten Blick keinen Grund hatte, das Sowjetsystem abzulehnen. ${ }^{29}$ Umgekehrt gibt es aber auch Beispiele von Personen, die aufgrund ihrer Repressionserfahrung ausreichenden Anlass gehabt hätten, die Sowjetmacht zu hassen und sich dennoch für den Untergrund engagierten.

Die Motivlagen für die Zusammenarbeit mit den Deutschen waren sehr vielschichtig: Materielle Beweggründe waren, wie es scheint, mindestens ebenso wichtig wie politische oder nationale, vor allem die Tatsache, dass die Hilfspolizisten neben anderen Privilegien auch die gleichen Lebensmittelrationen wie die deutschen Militärangehörigen erhielten. Die Hilfspolizisten waren für die Deutschen auch häufig kein besonders zuverlässiger Partner, sondern wechselten schnell wieder die Fronten, sobald sich die Lage an der Front veränderte.

Auch das bis heute verbreitete Stereotyp von den Ukrainern als Antisemiten wird von den Gerichtsakten und biographischen Schilderungen nur allzu oft revidiert: So waren es nicht nur Ukrainer, die in den Reihen der Hilfspolizei an den schrecklichsten Verbrechen der Deutschen gegen Juden partizipierten oder an Pogromen teilnahmen, sondern ebenso Russen und andere ethnische Minderheiten. Und Ukrainer waren ebenso wie andere Nationalitäten auch unter denjenigen, die Juden vor der Vernichtung durch die Deutschen retteten. Ein eindrucksvolles Beispiel, das die Komplexität der Kriegsbiographien verdeutlicht, ist das Schicksal der Margarita Evstaf evna Ševčenko aus Donec'k. Ihre jüdische Mutter war kurz nach der Besatzung der Stadt von den Deutschen ermordet worden. Ihr Vater, ein weißrussischer Bergbauspezialist, half den Deutschen beim Wiederaufbau der Bergbaubetriebe und wurde nach dem Krieg von einem sowjetischen Militärtribunal als „Vaterlandsverräter" verurteilt. Ihr Überleben verdanken Margarita Ševčenko und ihr jüngerer Bruder

27 Vgl. zur Kollaboration im Donbas: TANjA Penter Die lokale Gesellschaft im Donbass unter deutscher Okkupation 1941-1943, in: Beiträge zur Geschichte des Nationalsozialismus, Bd. 19: Kooperation und Verbrechen. Formen der „Kollaboration“ im östlichen Europa 1939-1945, 2003, S. 183223.

28 Vgl. Bericht über die Arbeit der Parteiorganisation im Gebiet Stalino, in: CDAHOU (Staatsarchiv der Höheren Staats- und Verwaltungsorgane der Ukraine), F. 1, Op. 23, D. 60, S. 1-24, hier S. 1, 12.

29 Vgl. KuromiYa Freedom, S. 283 f. 
vor allem ihrem ukrainischen Kindermädchen, einer einfachen Frau vom Dorf, die sich in den Kriegsjahren und danach aufopfernd um sie kümmerte. ${ }^{30}$ Auf der anderen Seite gab es auch Juden, die mit den Deutschen zusammenarbeiteten, an Verbrechen teilnahmen und in der Nachkriegszeit von sowjetischen Militärtribunalen als Kollaborateure verurteilt wurden. ${ }^{31}$

Noch bedeutsamer ist jedoch die Erkenntnis, dass sich all diese Phänomene in den Kriegsbiographien der Menschen vermischten, so dass Opfer zu anderen Zeitpunkten auch zu Tätern werden konnten, Kollaborateure zu Widerstandskämpfern und umgekehrt. Aus den Gerichtsakten ist ersichtlich, dass unter den Mitgliedern der Hilfspolizei viele Kriegsgefangene waren, die auf diese Weise der entsetzlichen Situation in den Kriegsgefangenenlagern zu entkommen suchten. Dies traf insbesondere auf Travniki-Männer, wie John (Ivan) Demjanjuk, der jüngst in München vor Gericht stand, zu. Ebenso häufig trifft man auf Fälle, in denen sich Sowjetbürger, die mit den Deutschen zusammengearbeitet hatten im Anschluss den Partisanen anschlossen. All dies zeigt, dass die von den Historikern bisher verwendeten abstrakten Kategorien für ein Verständnis der Gesellschaften im Krieg wenig geeignet sind. Häufig existierten keine klaren Trennlinien zwischen „Kollaboration” oder „Loyalität”, sondern die Handlungen der Menschen bewegten sich eher in moralischen Grauzonen. Phänomene wie Kollaboration, Widerstand oder auch Zwangsarbeit waren de facto also viel stärker miteinander verbunden und nicht statisch, sondern viel dynamischer als Historiker bisher glaubten.

$\mathrm{Zu}$ ähnlichen Befunden kann man auch im Zusammenhang mit dem letzten deutschen Wiedergutmachungsprogramm für ehemalige Zwangsarbeiter in der Ukraine kommen: Auch hier zeigte sich anhand von Hunderttausenden von Beschreibungen von Einzelschicksalen die Vielzahl und Komplexität der Kriegsbiographien, die eindeutige Kategorisierungen, wie sie sich die „Architekten“ des Entschädigungsgesetzes wünschten, häufig schwierig machten. So ließ sich beispielsweise der im Entschädigungsgesetz vorgesehene Tatbestand der Deportation nicht immer klar nachzeichnen, da insbesondere gegen Kriegsende die Grenzen zwischen Deportation, Vertreibung oder Flucht zunehmend fließend waren. Zudem stellte die Trennung zwischen gegen ihren Willen deportierten Zwangsarbeitern und „freiwilligen" Kollaborateuren die Entschädigungsbürokratien vor Definitionsprobleme. All dies zeigt, dass Versuche, im Nachhinein klare, (oftmals moralische) Trennlinien zu ziehen, dem realen Kriegs- und Besatzungsalltag nicht gerecht werden. Die vielschichtigen politischen, gesellschaftlichen und erinnerungspolitischen Auswirkungen des letzten Auszahlungsprogramms für ehemalige Zwangsarbeiter untersucht ein internationales Forschungsprojekt am Lehrstuhl für Zeitgeschichte an der Ruhr-Universität Bochum. In diesem Zusammenhang erstellt Julia Landau eine Studie zur Zwangsarbeiterentschädigung in der Ukraine und zur Rolle der nationalen ukrainischen Stiftung.

30 Vgl. Interview mit Margarita Evstafevna Ševčenko im Dezember 2003 in Donec'k.

31 Vgl. Penter Local Collaborators on Trial. 


\section{Ausblick}

Ein positives Ergebnis der deutschen Entschädigungsprogramme für ehemalige Zwangsarbeiter besteht darin, dass diese eine eigene spezifische Überlieferung hervorgebracht haben, die Historikern in der Zukunft zur vielfältigen Auswertung zur Verfügung steht. Dazu zählen umfangreiche Datenbanken über die ehemaligen Zwangsarbeiter, auf deren Grundlage sich deren Migrationswege in der Kriegs- und Nachkriegszeit, Familienmuster, soziale Lagen und anderes empirisch auswerten lassen. Bereits während des Auszahlungsprogramms konnten aus den Zeitzeugenberichten zahlreiche neue Erkenntnisse über Lager, Ghettos und andere Haftstätten in den besetzten Gebieten gezogen werden.

Einen besonders spannenden Quellenkorpus stellen zudem Briefe dar. Im Zuge des letzten Auszahlungsprogramms sowie des Vorläuferprogramms sind bei den nationalen Entschädigungsstiftungen seit Anfang der 1990er Jahre Hunderttausende von Briefen, darunter neutrale Anfragen, Beschwerden oder auch (eine kleine Zahl von Danksagungen) von NS-Opfern, eingegangen. Umfang und Gestaltung dieser Briefe sind sehr unterschiedlich: Manche umfassen nur wenige Zeilen, andere führen auf 30 Seiten und mehr den gesamten Lebensweg des Schreibers aus. Unter den Schreibenden sind alle sozialen Schichten und Bildungsgruppen repräsentiert - auch diese Tatsache erhöht den Wert dieser Quelle für den Historiker erheblich. In den Briefen zeigen sich die Komplexität der individuellen Kriegsbiographien sowie die vielfältigen Selbst- und Fremdwahrnehmungen der NS-Opfer und das Bild von den Deutschen. Darüber hinaus spiegeln die Briefe, die die ersten 15 Jahre des Systemwechsels nach dem Zusammenbruch der Sowjetunion erfassen, wider, wie diese Generation der Kriegsteilnehmer, die zu den Hauptverlierern des Systemwechsels nach dem Zusammenbruch der Sowjetunion zählen, die allgemeinen politischen, wirtschaftlichen und sozialen Transformationsprozesse erlebte und welche Vorstellungen von einer gerechten Gesellschaft und vom neuen Staatsaufbau sie entwickelte. 


\section{Weiterführende Literatur}

KAREL C. BERKHOFF Harvest of Despair. Life and Death in Ukraine Under Nazi Rule. Cambridge, Mass., London 2004.

RAy Brandon, Wendy Lower (Hg.) The Shoah in Ukraine. History, Testimony, Memorialization. Indiana 2008.

MARTIN DEAN Collaboration in the Holocaust. Crimes of the Local Police in Belorussia and Ukraine, 1941-44. New York 2000.

Handbuch der Lager, Gefängnisse und Ghettos auf dem besetzten Territorium der Ukraine (1941-194). Kyjiv 2000.

TANJA PENTER Kohle für Stalin und Hitler. Arbeiten und Leben im Donbass 1929 bis 1953. Essen 2010.

AMIR WEINER Making Sense of War. The Second World War and the Bolshevik Revolution. Princeton 2001.

Dieter PoHL Die Herrschaft der Wehrmacht. Deutsche Militärbesatzung und einheimische Bevölkerung in der Sowjetunion 1941-1944. München 2008.

DERS. Nationalsozialistische Judenverfolgung in Ostgalizien 1941-1944. München 1996. 